\section{Ophthalmic and visual profile of guide dog owners in Scotland}

K Refson, A J Jackson, A E Dusoir, D B Archer
Royal Victoria Hospital, Belfast, Queens University, Belfast

K Refson

A J Jackson

D B Archer

University of Ulster, Jordanstown, Northern Ireland

A E Dusoir

Correspondence to:

K Refson, Low Vision Unit, Ophthalmology Directorate, Royal Victoria Hospital, Belfast BT12 6BA.

Accepted for publication 10 November 1998

\begin{abstract}
Backgroundlaims-Out of an estimated 90000 visually impaired people in Scotland, 509 make use of a guide dog. Initial research in Northern Ireland suggests that the ophthalmic profile of guide dog owners (GDOs) is highly specific. The aim of this study was to compare the ophthalmic and visual characteristics of Scottish GDOs with other groups of visually impaired people.

Methods-A random sample of GDOs from central and northern Scotland $(n=82)$ underwent a detailed assessment of residual vision (distance and near acuity, visual fields, contrast, and glare sensitivity). Comparative data were obtained from two populations of visually impaired non-GDOs-one group attending hospital ophthalmic and low vision clinics $(n=50)$ the other social services rehabilitation clients $(n=35)$. All participants completed a questionnaire to elicit ophthalmic history, age, and registration details.
\end{abstract}

Results-GDOs were found to be significantly younger and more profoundly visually impaired than non-GDOs. The main causes of visual impairment were retinitis pigmentosa $(23 \%)$, optic atrophy $(15 \%)$, and retinopathy of prematurity $(7 \%)$. Ninety nine per cent of GDOs were registered blind and had been visually impaired for an average of 39 years. Only $31 \%$ were totally blind.

Conclusion-GDOs represent a unique minority of the visually impaired population. Epidemiological registration trends would suggest that the numbers of young profoundly visually impaired people are unlikely to increase relative to their elderly counterparts. This has implications on the future demand for guide dog ownership.

(Br F Ophthalmol 1999;83:470-477)

National blind and partially sighted registration figures identify $0.6 \%$ of the population of the United Kingdom as visually impaired. In Scotland, registered visual impairment is proportionately in line with national figures at almost 31000 people. ${ }^{1}$ However, it is known that official registration figures underestimate the prevalence of blindness and partial sight ${ }^{2}$ and epidemiological studies indicate that the true prevalence of visual impairment is almost twice that identified through registration. ${ }^{34}$ Community based surveys by Bruce et al in 1991 for the Royal National Institute for the Blind (RNIB) suggest there are approximately three times more blind and partially sighted people in the United Kingdom than identified in official figures. ${ }^{5}$ According to these estimates, over one million residents of Great Britain and Northern Ireland are eligible for registration. In contrast with these figures only 4600 visually impaired people make use of a guide dog with just over 500 of these resident in Scotland. ${ }^{6}$

A previous survey carried out in Northern Ireland by Jackson et al in 1994 indicated that guide dog owners represent a highly selective subgroup of the visually impaired population. Significant differences in age, health, visual functions, and ocular pathology were found compared with other visually impaired people. ${ }^{7}$ The study concluded that with an increasing prevalence of age related ocular disease guide dog owners, who tended to be younger, fitter, and more mobile than other visually impaired people, may form a smaller proportion of the registered blind in the medium to long term future. Should these findings be applicable to the UK and other developed countries, there are obvious repercussions for those involved with the planning and resource development of guide dog and mobility services for the visually impaired. There is, however, a paucity of previous research on the characteristics of those who currently successfully use guide dogs. The aim of this study was to determine if the ophthalmic, visual, health, and social characteristics of guide dog owners in two other regions of the UK differ from those of Northern Irish guide dog owners. This paper presents the ophthalmic and visual characteristics of guide dog owners in Scotland and compares these with data collected from two other groups of visually impaired people resident in Scotland. Adequate funding enabled us to expand on the information collected in the initial study while applying the same criteria as adopted in the Northern Ireland study.

Subjects and methods

The target population consisted of all guide dog owners (GDOs) resident in Scotland. For logistical reasons those living in extreme rural locations were excluded, leaving 390 GDOs in central and northern Scotland who were asked by letter to participate in the study. A cut off period was set at 2 weeks after which time 133 replies had been received. From this number 100 owners were randomly selected for assessment. Data were obtained on the age and sex distribution of non-responders from Guide Dogs for the Blind Association (GDBA) records in order to check the validity of the sample. 
Two other subgroups of visually impaired people (VIPs) were chosen as comparison populations: (1) hospital patients who were attending low vision clinics and registerable people from ophthalmic outpatient clinics, henceforth known as the "hospital" group; (2) VIPs who had received rehabilitation services from GDBA but had not applied for a guide dog (the "rehab" group). These were chosen on the grounds of being major subgroups within the visually impaired population who were easily identifiable and accessible through hospital clinics or from a central register. No attempt was made to age and sex match control populations as our initial objective was to determine whether the general profiles of individuals availing themselves of these services were fundamentally different. Data were obtained from 50 consecutive consenting patients attending low vision and ophthalmic clinics at Glasgow Eye Infirmary and St John's Hospital at Livingstone. Data were collected on two separate visits in July and October 1997. Criteria for those selected from ophthalmic clinics were vision of a level eligible for blind or partial sight registration and aged 16 or over. The second comparison group $(n=35)$ was obtained from the client base of social services visual impairment units in Dundee and Dumfries and Galloway who were under GDBA rehabilitation services contract.

The study method consisted of analysis of residual visual functions and a detailed questionnaire which was presented verbally to each participant in order to obtain information concerning ophthalmic history, general health, social, educational, and employment profiles. The questionnaire collected essentially the same information as that recorded in the 1994 Northern Ireland study. Additional questions relating to independence, mobility, psychological adjustment to visual impairment, and the benefits/drawbacks of guide dog use were included. This paper concentrates on the visual and ophthalmic findings. Other key results on guide dog ownership will be referred to here but the full extent of the health, social, and psychological data analysis will appear in a second paper. ${ }^{8}$ A subjective assessment of vision, with a ranked score, was added to the questionnaire. This assessment comprised the following questions: "Can you see a window in a room during daylight? Can you see shapes of furniture in a room? Can you recognise a person's features at arm's length, across a room, across a street?" Questions were numbered from 1 to 5 and the score given was the number of the last question which was answered in the affirmative. If the answer to question 1 was given as "no" it was given a zero score.

A total of 82 out of the 100 GDOs completed the assessment and interview, the remaining 18 did not attend owing to sickness or holidays. All tests and interviews were carried out by the first author thus minimising observer bias. The following tests were carried out on all participating GDOs: refraction, distance visual acuities (Snellen and SonksenSilver charts), near visual acuities (BaileyLovie charts at $25 \mathrm{~cm}$ with a +4.00 DS spectacle addition), contrast sensitivity (PelliRobson chart at 1 metre), ${ }^{9}{ }^{10}$ glare sensitivity (Mentor Brightness Acuity Test), ${ }^{112}$ and assessment of colour vision with the City University test and Munsell D15 test with $2.5 \mathrm{~cm}$ caps (PV16 test from Precision Vision, USA). Visual fields were assessed by confrontation using three white targets: $1 \mathrm{~cm}, 1.5 \mathrm{~cm}$, and 2.5 $\mathrm{cm}$ in diameter. A monocular assessment was made using the smallest perceivable target which was brought from periphery to centre in an arc $30 \mathrm{~cm}$ in radius from the test eye. Fields were then classified into five categories according to the extent of the binocular field loss (where $1=$ full field to $5=$ almost complete visual field loss). Conventional perimetry was not feasible since equipment needed to be portable and would be unsuitable for those with profound visual loss. Ophthalmoscopy was carried out to confirm diagnosis of ocular pathology.

Visual assessments of GDOs took place at regional GDBA centres under controlled conditions. Visual acuity and contrast sensitivity charts were externally illuminated at a luminance of 1000 lux, and room illuminance measured 450 lux. Rehab clients, who were seen at their local social services office and other locations, were assessed under similarly controlled conditions. With the hospital group measurements of corrected Snellen distance VA were taken by low vision clinic optometrists, all of whom were familiar with the study protocol, immediately before their interview. Additional diagnostic information was available from the hospital notes. All other tests (with the exception of contrast and glare sensitivity, which because of practical reasons and time constraints could not be carried out) were performed by the first author under the same conditions used for the GDOs.

\section{STATISTICAL METHODS}

For the purposes of statistical analysis Snellen acuities were converted into equivalent values of visual angle using the decimal scale. Where VA was less than $0.5 / 60$, the resolution distances of the largest Snellen letter were measured and converted into decimal notation. This avoided the inaccuracies of the terms hand movements (HM) and counting fingers (CF). Perception of light (PL) was given the arbitrary decimal value of 0.0005 . Nonparametrical statistical tests were used to analyse results throughout since much of the data are ordinal or nominal and the interval data are not normally distributed. Comparisons of the three populations were made using the Kruskal-Wallis and $\chi^{2}$ test and, where two medians were compared, the Mann-Whitney test. Kendall's tau correlation coefficient tested for correlation between variables.

\section{Results}

AGE AND SEX

Table 1 gives the summary statistics and Figure 1 compares the age distributions of the three groups. The guide dog owners were found to be a significantly younger population $(\mathrm{H}=55$, $\mathrm{p}<10^{-11}$ ) with a mean age difference of 19 years 
Table 1 Age and sex distribution of the three groups

\begin{tabular}{llllll}
\hline & $\begin{array}{l}\text { GDOs } \\
(n=82)\end{array}$ & $\begin{array}{l}\text { Hospital } \\
\text { group }(n=50)\end{array}$ & $\begin{array}{l}\text { Rehab group } \\
(n=35)\end{array}$ & Test statistic & $p$ Value \\
\hline $\begin{array}{lllll}\text { Age: } \\
\text { mean }\end{array}$ & 54.1 & 73.4 & 73.3 & Kruskal-Wallis & \\
SD & 15.6 & 12.8 & 14.1 & $\mathrm{H}=55$ & $<10^{-11}$ \\
$\quad$ range & $23-81$ & $19-94$ & $38-92$ & & \\
Sex: & & & & & \\
$\quad$ males & 40 & 14 & 10 & $\chi^{2} 2 \mathrm{df}$ & \multirow{2}{*}{0.02} \\
$\quad$ females & 42 & 36 & 25 & & \\
M:F ratio & $1: 1.1$ & $1: 2.6$ & $1: 2.5$ & 7.46 & \\
\hline
\end{tabular}

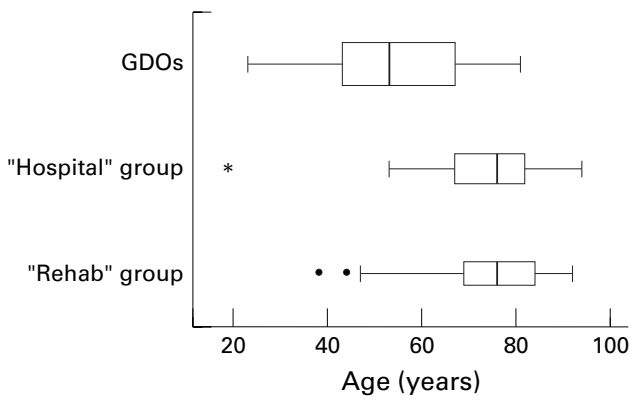

Figure 1 Schematic plot of the age distribution of the three groups. The schematic plot conventions were as follows: the box vertical edges give the lower quartile, median, and upper quartile; "outliers" are plotted individually, as a dot if they lie between $11 / 2$ and 3 interquartile ranges outside the quartiles, as a star if they lie more than 3 interquartile ranges outside the quartiles; the whiskers go out to the most extreme non-outlying observations.

less than either of the other two groups of visually impaired people. Male to female ratios of GDOs were more than twice those of the comparison populations. Whereas the GDOs sample comprised almost equal numbers of males and females, at least two and a half times as many females made up the comparison groups as males. This is in line with age matched blind registration figures ${ }^{13}$ and is a reflection of the increased longevity of females in the normal population. There were no significant differences in the age and sex profiles of the two comparison populations. Data were available on the age and sex distribution of all 507 Scottish guide dog owners: the mean age was 52.8 years with a range from 19 to 88 . The whole population consisted of 277 males and 230 females giving a male to female ratio of 1:0.8. There were no significant differences from our sample population in either age distribution $\left(\chi^{2}=76, \mathrm{df}=67, \mathrm{p}=0.2\right)$ or male to female ratios $\left(\chi^{2}=1.35, \mathrm{df}=1, \mathrm{p}=0.2\right)$.

REGISTRATION AND ONSET OF VISUAL

IMPAIRMENT

The three populations of VIPs differed significantly in their registration status $\left(\chi^{2}=90.8\right.$, $\mathrm{p}<10^{-15}$ ) (Table 2). GDOs had a $100 \%$ registration rate and all but one were registered as blind. The rehabilitation clients were almost

Table 2 Registration category and length of registration

\begin{tabular}{llll}
\hline Registration & GDOs $(n=82)$ & Hospital $(n=50)$ & Rehab $(n=35)$ \\
\hline Blind & $99 \%(81)$ & $30 \%(15)$ & $66 \%(23)$ \\
Partially sighted & $1 \%(1)$ & $24 \%(12)$ & $31 \%(11)$ \\
Not registered & 0 & $46 \%(23)$ & $3 \%(1)$ \\
Years registered & & & \\
$\quad$ median & 22 & 0.25 & 1.0 \\
min & 3 & 1 month & 1 month \\
max & 80 & 25 & 30 \\
\hline
\end{tabular}

${ }^{\star}$ Number of years on the blind register, or if not registered as blind, the partial sight register.



Figure 2 Age at onset of visual impairment.

all registered but the proportion of partially sighted people increased to one third. Those attending hospital clinics were less likely to be registered and only $30 \%$ were registered as blind.

GDOs were found to have been visually impaired from an early age with a mean duration of visual impairment of 39 years. They were registered for correspondingly long periods of time. A close correlation was found between the age of onset of visual impairment and the number of registered years $(\tau=-0.27$, $\mathrm{p}=0.0004$, one tail). In marked contrast, those who did not use a guide dog (non-GDOs) had suffered more recent visual impairment much later in life. The mean length of visual impairment was 7.6 and 7.7 years for the hospital and rehab groups respectively and their distribution of onset of visual impairment is shown in Figure 2. These differences were highly significant (Kruskall-Wallis $\mathrm{H}=102, \mathrm{p}<10^{-15}$ ).

There was no difference between hospital or rehab groups in the age of onset or duration of visual impairment; however, the rehab clients were registered for significantly longer periods of time (Mann-Whitney $\mathrm{U}=524, \mathrm{p}=0.0007$, one tail).

The GDOs had owned their guide dog from between 3 months to 45 years with a median of 10.5 years of guide dog ownership. The number of years of guide dog use correlated with the duration of visual impairment $(\tau=$ $0.37, \mathrm{p}<10^{-6}$ ) and more closely with the number of blind registered years $(\tau=0.54$, $\mathrm{p}<10^{-11}$, one tail). There was an average of 12.5 years between registration and obtaining the first guide dog.

\section{OCULAR PATHOLOGY}

The main ophthalmic conditions causing visual impairment are listed in Table 3. Guide dog owners notably suffered from a more diverse range of pathologies than the other subgroups, principally retinitis pigmentosa $(23 \%)$, optic atrophy (15\%), retinopathy of prematurity $(7 \%)$, and microphthalmos $(7 \%)$. In contrast, by far the most frequently occurring condition among the non-GDOs was age related macular degeneration (ARMD) which affected half of the hospital attenders and $40 \%$ of the rehab clients. Primary open angle glaucoma and diabetic retinopathy were identified as other major causes of visual loss common to the latter subgroups but only accounted for two cases each among the guide dog owners. 
Table 3 Ocular pathology

\begin{tabular}{lccc}
\hline & GDOs $(n=82)$ & Hospital $(n=50)$ & Rehab $(n=35)$ \\
\hline Retinitis pigmentosa & 19 & 0 & 0 \\
Optic atrophy & 12 & 0 & 3 \\
Retinopathy of prematurity & 6 & 0 & 0 \\
Microphthalmos & 6 & 0 & 0 \\
Congenital cataract & 5 & 0 & 0 \\
Optic neuritis & 4 & 2 & 0 \\
Hereditary retinal dystrophy & 4 & 0 & 0 \\
Retinal detachment & 3 & 4 & 3 \\
Uveitis & 3 & 0 & 0 \\
Ocular trauma & 3 & 0 & 0 \\
Congenital glaucoma & 3 & 0 & 0 \\
Primary open angle glaucoma & 2 & 6 & 4 \\
ARMD & 2 & 25 & 14 \\
Diabetic retinopathy & 2 & 6 & 4 \\
Hemianopia (stroke) & 0 & 2 & 5 \\
Others & 8 & 5 & 2 \\
\hline
\end{tabular}

Table 4 Aetiological classification of pathology

\begin{tabular}{lrlrlrl}
\hline & GDOs & \multicolumn{3}{c}{ Hospital } & Rehab & \\
\hline Degenerative & $43 \%$ & $(35)$ & $82 \%$ & $(41)$ & $80 \%$ & $(28)$ \\
Congenital & $33 \%$ & $(27)$ & $2 \%$ & $(1)$ & $0 \%$ & $(0)$ \\
Inflammatory & $11 \%$ & $(9)$ & $0 \%$ & $(0)$ & $0 \%$ & $(0)$ \\
Traumatic & $7 \%$ & $(6)$ & $2 \%$ & $(1)$ & $3 \%$ & $(1)$ \\
Neoplastic & $4 \%$ & $(3)$ & $0 \%$ & $(0)$ & $6 \%$ & $(2)$ \\
Metabolic & $2 \%$ & $(2)$ & $14 \%$ & $(7)$ & $11 \%$ & $(4)$ \\
Total & $100 \%$ & $(82)$ & $100 \%$ & $(50)$ & $100 \%$ & $(35)$ \\
\hline
\end{tabular}

Ocular pathology was further classified into the aetiological groups shown in Table 4 which revealed highly significant differences between the subpopulations $\left(\chi^{2}=55.2, \mathrm{df}=10, \mathrm{p}<10^{-7}\right)$. Degenerative conditions accounted for at least $80 \%$ of the pathology of both comparison groups but this figure was almost halved for GDOs. Congenital eye disorders accounted for one third of causes of blindness among GDOs and inflammatory eye disease also featured significantly. Hospital attenders and rehab clients showed a similar pattern of pathology and there was no significant difference in aetiological classification between these two subgroups at the $5 \%$ level $(\mathrm{p}=0.1)$.

Classification of pathology into primary site of disease increased the significance of these discrepancies $\left(\chi^{2}=68.7, \mathrm{df}=14, \mathrm{p}<10^{-8}\right)$. Retinal disease (excluding primary maculopathies) accounted for $46 \%$ of visual disorders among GDOs but only $22 \%$ and $20 \%$ of hospital and rehab patients, respectively. The optic nerve was the second most affected site of pathological damage for GDOs (19.5\%) and affected a slightly lesser proportion of people in the other subgroups (16\%, $17 \%$ respectively). Almost one third of guide dog owners (25/82) were currently under review at ophthalmology outpatient clinics.

\section{VISUAL FUNCTIONS}

Visual acuity (VA) was recorded as the optimum acuity in the better eye after refraction and converted to decimal notation. Guide dog owners recorded significantly poorer visual acuities than either of the comparison subgroups $\left(\mathrm{H}=66.6, \mathrm{p}<10^{-14}\right)$. As can be seen from the distribution of visual acuities of the three sample groups in Figure 3, the hospital subgroup obtained the best VA scores, significantly higher than the rehab group $(\mathrm{p}=0.004)$. Seventy out of the 82 GDOs surveyed recorded visual acuities of $3 / 60$ or less in the better eye, which is the recommendation given

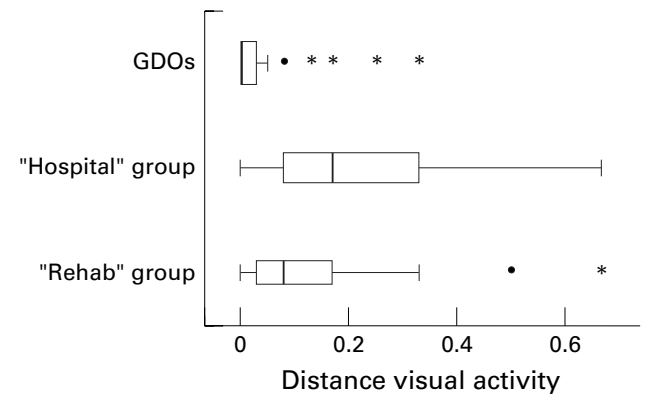

Figure 3 Distance visual acuity, decimal notation.

for registration as blind on the BD8 certification form (BP1 in Scotland). Just under half of those in the rehab group (17/35) and only one fifth $(10 / 50)$ of the hospital group had VA of this level. Twenty five GDOs (30.5\%) were totally blind (no perception of light) whereas only one non-GDO, who was in the rehab group, had no light perception.

Near VA was similarly distributed with the GDOs having the poorest, and hospital patients the best near acuity. Differences were significant at $10^{-6} \%$ level. The range of acuities for all three groups was wide, between $\mathrm{N} 5$ to N80 (for those above PL). The majority of the comparison subgroups ( $82 \%$ hospital, $60 \%$ rehab), but only $28 \%$ of GDOs could read print of $\mathrm{N} 50$ or smaller. Interestingly, in spite of such poor near vision, 30 GDOs (37\%) were making effective use of low visual aids (LVA). LVA use was higher in the rehab group where the figure rose to $69 \%$.

Results from the subjective assessment of residual vision showed that $49 \%$ of GDOs, $91 \%$ of rehab clients, and $100 \%$ of hospital patients felt they had some useful remaining vision as opposed to no useful vision. The ordinal subjective vision scores from the GDOs were correlated with objective distance and near acuity measurements. A significant association was found between both distance VA $(\tau=$ $0.6, \mathrm{p}<10^{-10}$, one tail) and log near VA $(\tau$ $\left.=-0.5, \mathrm{p}<10^{-7}\right)$.

\section{Visual fields}

The majority of GDOs had severely constricted visual fields (VF). A measurable field was obtained from 51 GDOs, the remainder having insufficient residual vision. Of these, $85 \%$ had lost three quarters or more of their binocular field (Table 5). While the differences between GDOs and the comparison populations were highly significant $\left(\mathrm{H}=57.9, \mathrm{p}<10^{-12}\right)$, the distribution of visual field classifications in the hospital and rehab groups is virtually identical (Fig 4), with the majority retaining more than half of their binocular field.

\section{Contrast sensitivity and glare sensitivity}

Only 30 out of the 82 GDOs retained enough residual vision to enable measurement of contrast sensitivity (CS) with the Pelli-Robson chart at 1 metre. CS was reduced by at least $1.2 \log$ units in 29 of these cases (98\%). This degree of CS loss is considered clinically significant. ${ }^{14}$

Sensitivity to glare was high among the GDOs: 28 out of 30 with VA of at least 1/60 
Table 5 Visual field classifications

\begin{tabular}{llccc}
\hline Visual field grading & Extent of binocular field & GDO & Hospital & Rehab \\
\hline 1 & full & 0 & 5 & 2 \\
2 & $>$ half & 4 & 17 & 12 \\
3 & $1 / 4-1 / 2$ & 4 & 9 & 6 \\
4 & $<1 / 4$ & 7 & 6 & 4 \\
5 & isolated points & 36 & 12 & 8 \\
0 & nil & 31 & 1 & 3 \\
Total & & 82 & 50 & 35 \\
\hline
\end{tabular}

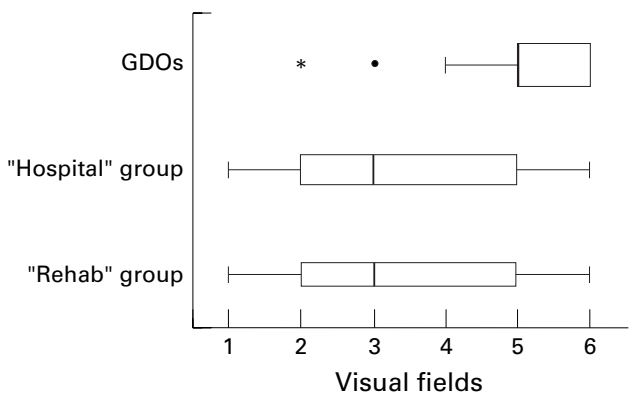

Figure 4 Visual field gradings. $1=$ full field, $5=$ profound field loss. 6 = no measurable field.

were able to undergo the brightness acuity test (BAT).${ }^{112}{ }^{12}$ Seventy one per cent were found to suffer a "significant" reduction in VA with the BAT at $100 \mathrm{ft}$ lamberts (mid-setting: the manufacturers equate this to conditions of "indirect sunlight"). A "significant" reduction in VA was defined as a drop in VA between 0.3 and $0.5 \mathrm{log}$ units. Insufficient data were collected from the comparison groups for these functional measures of vision owing to clinical time constraints and a reluctance on the part of some patients to undergo glare testing. It was felt that more useful data on the complete range of ophthalmic, health, and social status questions would be gained from interviewing as many people willing to participate as possible rather than restricting numbers to only those who could undertake the full visual assessment.

\section{Colour vision}

One third of the GDOs were able to undergo an assessment with the City University test (TCU). Seven obtained entirely normal scores and a further seven identified as normal at least four out of the six tests at "chroma four" size. The remaining 13 demonstrated colour anomalies - one deutan, two tritan, five mixed anomalies, and five with no colour discrimination. Approximately half of the GDOs with sufficient residual vision to undertake the TCU test, therefore, exhibited normal colour perception. A more appropriate test for use with low vision patients, a large field modified Munsell D15 (PV16 test from Precision Vision) only became available towards the end of the study. Of 14 GDOs assessed, nine had sufficient residual vision to perform the test. Six exhibited normal colour vision with all the caps arranged in the correct order, two showed a deutan defect, and one a mixed anomaly. The PV16 test revealed additional information over the TCU in five cases. Two exhibited a normal result where the TCU gave a mixed colour anomaly. Of the three who could not see the TCU plates, two subjects were shown to have a deutan defect and one a mixed colour deficiency. Preliminary use suggests that the numbers able to undergo colour vision assessment would be increased with this test and diagnosis of colour anomaly made less ambiguous. Colour contrast is frequently used to aid visibility of potential dangers such as steps and railings. Colour contrast is also used to highlight objects used in daily living tasks undertaken by the visually impaired. This approach can still be justified for use with GDOs with profound visual impairments as two thirds of those who felt they had useful remaining vision (one sixth of total GDOs) showed normal colour perception and a further $10 \%$ could distinguish bright primary colours. The GDOs were also asked if they had a preference for any particular colour used to mark items: the favourite was yellow closely followed by white. These are the most commonly used background colours to achieve contrast enhancement on signs and symbols. Recommendations from the RNIB/GDBA Joint Mobility Unit for contrast enhancing colours have recently been changed to specify luminance contrast rather than advising on a particular object or surround colour. Twelve GDOs who failed the colour vision tests felt they could see fluorescent yellow or other bright colour. It is apparent that luminance contrast and reflectance are the reasons why this helps in identifying objects rather than colour discrimination.

Although $49 \%$ of GDOs described their residual vision as being useful and the same percentage recorded acuities above PL, it is apparent that their functional vision is extremely limited. In spite of this, however, almost half (48\%) still wore spectacles and one third of these continued to wear their spectacles to improve acuity (the remaining two thirds wore spectacles to reduce glare or light levels, for cosmetic reasons, for protection or out of habit). This would indicate that significant numbers of GDOs require regular refractions, and even more require continued low vision assessments. These findings contradict the commonly held misconception that all guide dog users are totally blind and therefore the temptation to regard further optometric assistance as unnecessary.

\section{Discussion}

The results of this study confirm the findings of Jackson et al that guide dog owners represent a relatively younger and more profoundly visually impaired subgroup in comparison with other visually impaired populations. ${ }^{7}$ GDOs from both Scotland and Northern Ireland generally suffer visual impairment from an early age whereas the trend for those who make use of other rehabilitation and low vision services is for recent loss of vision later in life. The major differences between the GDOs and non-GDOs stem from the contrasting nature of ophthalmic pathology. For the GDOs, ophthalmic conditions tended to be early onset degenerative or congenital, in contrast with mainly age related ocular disorders seen in the comparison groups. Virtually all GDOs in both studies are 
not only registered blind but have been on the register for long periods of time. Rehabilitation clients and low vision aid users, on the other hand, show a pattern of registration in keeping with their late onset and less severe visual impairment. Although registration is not a prerequisite to using a guide dog, there is a strong correlation between length of registration and duration of guide dog ownership and registration preceded guide dog ownership in every case. Registration may be the first stage in acceptance of irreversible disability and a recognition that other new strategies will need to be adopted to optimise quality of life. Acceptance was found to be correlated with duration of visual impairment. ${ }^{8}$ Those with recent onset conditions may be expected to be less well adjusted and have a poorer acceptance of their visual impairment which was indeed found to be the case. However, the idea of obtaining a guide dog had been rejected by the vast majority of both hospital and rehab participants irrespective of the length of their visual impairment. The grounds for rejection were predominantly sight being sufficient for present needs, a dislike of dogs, and advanced age.

The sample of GDOs participated voluntarily, therefore, there is a possibility that they are not entirely representative of the whole population. Available data on the age and sex of nonrespondents confirmed no significant difference in these variables from the sample population $(\mathrm{p}>0.2)$. Furthermore, the similarity of all results to the Northern Ireland population of guide dog owners suggests that our sample is representative of Scottish GDOs as a whole.

Guide dog owners were found to have severe limitations in all areas of visual function, nevertheless, those in Scotland would appear to have more residual vision than GDOs in Northern Ireland. Thirty seven per cent of Scottish GDOs surveyed were regularly using LVAs as opposed to only $5 \%$ of those in Northern Ireland, and $49 \%$ in Scotland felt they had some useful residual vision against only $27 \%$ in Northern Ireland. Scottish GDOs recorded better VA measurements than those in the Northern Ireland study. However, it is recognised that these results are not directly comparable since measurements of VA were recorded retrospectively from hospital notes in Northern Ireland with a possible lesser degree of accuracy. A review of the Northern Irish data is currently under way in order to confirm or deny these findings. In the current study, ranked scores of subjective appreciation of vision were found to correlate highly with objective measures of both near and distance acuity $(\tau=0.6, \mathrm{p}<0.0001)$. This indicates that useful information on the patients' visual status can be gained by asking simple questions and could be incorporated into a visual assessment by non-ophthalmic professionals such as social workers or rehab workers.

Given the nature of the three subgroups of VIPs who have differing, but overlapping, needs (that is, GDOs whose primary requirement is improved mobility, LVA users whose assistance is with a central acuity task, and rehabilitation clients who were either unsuitable or not in favour of mobility assistance in the form of a guide dog) it would not be surprising to find a difference in their visual functions. The types of visual function most important to mobility performance have been investigated by several authors. ${ }^{15-17}$ Visual fields have been found to be a more important predictor of orientation and mobility performance than visual acuity. Contrast sensitivity and visual fields each have more influence on orientation mobility than does visual acuity, and the combined effects of CS and VF have an even stronger relation with mobility performance. ${ }^{16}{ }^{17}$ In particular, the central 37 degrees of visual field and lateral and inferior mid-peripheral zones were found by LovieKitchin et al to be the areas most important to mobility performance. ${ }^{18}$ The main types of ocular pathology among GDOs-namely, retinitis pigmentosa (RP) and optic atrophy, result in extensive restriction of the visual fields. In contrast, the main pathology affecting the LVA users and rehab group was ARMD resulting in central acuity loss. It has been reported anecdotally in the literature that people with ARMD and central field loss generally do not experience the same difficulties with mobility as those with peripheral visual field loss. ${ }^{19}$ One third of hospital patients and 20\% of rehab clients in fact did not require any assistance with mobility in contrast with the GDOs who (by definition) all used a mobility aid. Factors such as age, health, and confidence obviously influence mobility and we are hopeful that subsequent analysis of other data collected during the course of this study will provide additional useful information. Nevertheless, the results of the above quoted studies would lead to the assumption that the difference in nature of field loss between GDOs and non-GDOs is certainly significant in terms of mobility performance.

The above factors, peculiar to GDOsnamely, age, registration, visual and ophthalmic status, could be useful as predictors of suitable candidates for guide dog ownership. Optometric and medical practitioners should be alerted to mobility needs of their patients. Improved communication to rehabilitation workers as to the pathologies and residual visual functions of those who may benefit from mobility training or the use of a guide dog, would provide a more effective, integrated service to the visually impaired.

Also important in the suitability of people to become guide dog users are psychological and personality factors. Some psychological variables have been investigated in relation to orientation mobility performance ${ }^{20-22}$ but further work would be necessary with the addition of the guide dog and the associated multiple psychological variables. Heyes et al in 1996 surprisingly found no correlation between psychological variables, such as personality and intelligence, and mobility performance. For their group of RP subjects, vision was the primary determinate of functional mobility performance. ${ }^{23}$ 
With the exception of diabetic retinopathy, the causes of blindness among GDOs reflect the important causes in people of working age-namely, retinitis pigmentosa and optic atrophy. ${ }^{24}$ LVA users and rehab clients exhibited a pattern of pathology in keeping with general blind registration trends ${ }^{25}$ - principally, ARMD, glaucoma, and diabetic retinopathy. These have also been found to be the most common causes of visual handicap in Scotland ${ }^{26}{ }^{27}$ ARMD and diabetic retinopathy, two of the leading causes of blindness in the Western world, ${ }^{24}$ accounted for only two causes each of visual loss among the GDOs surveyed in Scotland and a similarly small proportion in Northern Ireland. While not surprising to find very few GDOs with ARMD, a condition which does not appear to limit mobility to any great extent, the low rate of diabetic retinopathy is more unexpected since this condition occurs most frequently in those of a comparable age group to GDOs and also causes peripheral visual loss. The most likely explanation is that those registered blind due to diabetic retinopathy suffer other health and mobility problems related to their diabetes. Conclusions from both Scottish and Northern Irish studies indicate that the visually impaired who choose to use guide dogs are unlikely to suffer primarily from age related degenerative ocular disease but are drawn from a younger subpopulation of the registered blind, principally affected by early onset, hereditary, and congenital conditions. The similarities of findings with the Northern Ireland study would appear to suggest that the ophthalmic characteristics of GDOs are not peculiar to individual regions but reflect a national trend. Further research is in progress in England to test this hypothesis.

It is useful to consider how the continuation of current trends in blind registrations may alter the demographic balance of the visually impaired population. While age standardised registration rates for all causes of blindness have decreased, registrations attributed to ARMD have increased by $30-40 \%$ in Britain over the past 40 years. ${ }^{25}$ Over this period the prevalence of retinopathy of prematurity has also decreased; from being the commonest cause of blindness in children in the 1940s and 1950 s, it now accounts for only $6-18 \%$ of childhood registered blindness in industrialised countries. ${ }^{28}$ Retinitis pigmentosa, which accounts for $22 \%$ of the primary pathology among GDOs, occurs at a rate of 1 in 5000 births ${ }^{29}$ but, given the current levels of research and continued genetic counselling, it would seem reasonable to assume that the prevalence will decline relative to aging causes of blindness. With the continuation of current trends, the elderly will make up an increasing proportion of the registered blind. The implications of this are a significant alteration in the age and ocular pathological condition of the potential guide dog owner. Results from both this and the Northern Ireland study suggest that guide dog ownership will feature less highly in the future blind population. This would have important consequences for those involved with the allocation of resources to rehabilitation, mobility, and guide dog services.

Guide dogs undoubtedly make a substantial difference to the independence and mobility of the severely visually impaired and, as such, strenuous efforts must be made to ensure that those who may benefit from guide dog ownership are not neglected. The above results identify the age, visual, and ophthalmic profile of current guide dog owners and as such provide valuable information which may assist the GDBA in targeting those who are most likely to benefit from a guide dog. Further research is, however, needed into the ophthalmic, social, and psychological characteristics of guide dog applicants with a view to determining the differences between those who are either deemed unsuitable, or alternatively reject the option, and those who ultimately become successful guide dog users.

The authors wish to thank the Guide Dogs for the Blind Association for their financial assistance with this study and kind help in coordinating contact with individual guide dog owners. The help is gratefully acknowledged of staff at the Glasgow Eye Infirmary and St John's Hospital at Livingstone, Glasgow Resource Centre, Dundee Society for the Visually Impaired, and Dumfries and Galloway Social Services Visual Impairment Unit. Particular thanks are due to all the guide dog owners, hospital patients, and rehabilitation clients who participated in the study.

1 Social Services Statistics (1996/97). London: Department of Health Statistics Division; DHSS (NI). Social Services Statistics for Northern Ireland, Belfast; The Scottish Office Social Work Services Group Statistics. Edinburgh; The Social Work Services Group Statistics. Edinburgh; The Services Statistics for Wales, Cardiff; Office for National Services Statistics for Wales, Cardiff; Office

Statistics, mid-year population estimates, UK.
Gibson JM, Lavery JR, Rosenthal AR. Blindness and partial sight in an elderly population. Brf Ophthalmol 1986;70: $700-5$.

3 Cullinan TR. Visually disabled people in the community. Health Services Research Unit Report 28. Canterbury: University of Kent at Canterbury, 1977.

4 Robinson R, Deutsch J, Jones HS, et al. Unrecognised and unregistered visual impairment. Br f Ophthalmol 1994;78: 736-40.

5 Bruce IW, McKennell AC, Walker EC. Blind and partially sighted adults in Britain. RNIB Survey Vol 1. London: HMSO, 1991

6 Guide Dogs for the Blind Association. Guide Dogs for the Blind Association monthly training and breeding report. Reading: GDBA, December 1997.

7 Jackson AJ, Murphy PJ, Dusoir T, et al. Ophthalmic, health and social profile of guide dog owners in Northern Ireland. Ophthalmol Physiol Opt 1994;14:371-7.

8 Refson K, Jackson AJ, Dusoir T, et al. The health and social status of guide dog owners and other visually impaired adults in Scotland. (In press)

9 Pelli DG, Robson JG, Wilkins AJ. The design of a new letter chart for measuring contrast sensitivity. Clin Vis Sci 1988;2:187-9.

10 Leat SJ, Lockley D. The validity of the Pelli-Robson contrast sensitivity chart and its use in low vision. Ophthal Physiol Opt 1990;10:411.

11 Holladay JT, Prager TC Trujillo J, et al. Brightness acuity test and outdoor visual acuity in cataract patients. $\mathcal{F}$ Cataract Refract Surg 1987;13:67-9.

12 Neumann AC, McCarty GR, Locke J, et al. Glare disability devices for cataractous eyes, a consumer's guide. $\mathcal{F}$ Cataract Refract Surg 1988;14:212-16.

13 Evans J. Causes of blindness in England and Wales 1990-1991. Evans J. Causes of blindness
London: HMSO, 1995.

14 Whittaker SG, Lovie-Kitchin JE. The assessment of contrast sensitivity and contrast reserve for reading rehabilitation. n: Kooijman AC, Looijestijn PL, Welling JA, van der Wildt GJ, eds. Low vision. Amsterdam: IOS Press, 1994:88-92.

15 Brown B, Brabyn JA. Mobility and low vision: a review. Clin Exp Optom 1987;70:96-101.

16 Marron JA, Bailey IL. Visual factors and orientationmobility performance. Am f Optom Physiol Opt 1982;59: $413-26$

17 Long RG, Rieser JJ, Hill EW. Mobility in individuals with moderate visual impairments. $\mathcal{F}$ Vis Impair Blindness 1990; 84:111-18.

18 Lovie-Kitchin J, Mainstone J, Robinson J, et al. What areas of the visual field are important for mobility in low vision of the visual field are important for mot
patients? Clin Vis Sci 1990;5:249-63.

19 Haymes SA, Guest DJ, Heyes AD, et al. The relationship of vision and psychological variables to the orientation and 
mobility of visually impaired persons. $\mathcal{F}$ Vis Impair Blindness 1996;90:314-24

20 White K, Carroll R, Martin W, Personality factors associated with the decision to accept or reject mobility training. $\mathcal{F}$ Vis Impair Blindness 1990;84:470-4.

21 Beggs WDA. Psychological correlates of walking speed in the visually impaired. Ergonomics 1991;34:91-102.

22 Dodds AG, Bailey P, Pearson A, et al. Psychological factors in acquired visual impairment: the development of ascale of adjustment. $\mathcal{F}$ Vis Impair Blindness 1991;85:30610.

23 Heyes T, Haymes S, Guest D, et al. It is residual vision, not personality, that is more important for successful mobility. ity Conference 8, 1996.
24 Foster A, Johnston GJ. Magnitude and causes of blindness in the developing world. Int Ophthalmol 1990;14:135-40. 5 Evans J, Wormold R. Is the incidence of age-related macula degeneration increasing? Br f Ophthalmol 1996;80:9-14.

26 Ghafour IM, Allan D, Foulds WS. Common causes of blindness and visual handicap in the west of Scotland. Br F Ophthalmol 1983;67:209-13.

27 Balatsoukas DD, Sioulis C, Parisi A, et al. Visual handicap in south-east Scotland. F $R$ Coll Surg Edinb 1995;40:4951

28 Gilbert C, Rahi J, Eckstein M, et al. Retinopathy of prematurity in middle income countries. Lancet 1997:350:12-14.

29 Bundey S, Crews SJ. A study of retinitis pigmentosa in the $417-28$ 